\title{
BCL2 inhibits cell adhesion, spreading, and motility by enhancing actin polymerization
}

\author{
Hengning Ke ${ }^{1}$, Vandy I Parron ${ }^{1}$, Jeff Reece ${ }^{2}$, Jennifer Y Zhang ${ }^{4}$, Steven K Akiyama ${ }^{3}$, John E French ${ }^{1}$ \\ ${ }^{1}$ Laboratory of Molecular Toxicology, National Institute of Environmental Health Sciences, Research Triangle Park, NC 27709, \\ USA; ${ }^{2}$ Laboratory of Signal Transduction, National Institute of Environmental Health Sciences, 111, TW Alexander Dr. MD K2- \\ 08, Research Triangle Park, NC 27709-2233, USA; ${ }^{3}$ Laboratory of Molecular Carcinogenesis, National Institute of Environmental \\ Health Sciences, Research Triangle Park, NC 27709, USA; ${ }^{4}$ Department of Dermatology, Duke University Medical Center, Dur- \\ ham, NC 27710, USA
}

BCL2 is best known as a multifunctional anti-apoptotic protein. However, little is known about its role in celladhesive and motility events. Here, we show that BCL2 may play a role in the regulation of cell adhesion, spreading, and motility. When BCL2 was overexpressed in cultured murine and human cell lines, cell spreading, adhesion, and motility were impaired. Consistent with these results, the loss of $B c l 2$ resulted in higher motility observed in Bcl2null mouse embryonic fibroblast (MEF) cells compared to wild type. The mechanism of BCL2 regulation of cell adhesion and motility may involve formation of a complex containing BCL2, actin, and gelsolin, which appears to functionally decrease the severing activity of gelsolin. We have observed that the lysate from MCF-7 and NIH3T3 cells that overexpressed BCL2 enhanced actin polymerization in cell-free in vitro assays. Confocal immunofluorescent localization of BCL2 and F-actin during spreading consistently showed that increased expression of BCL2 resulted in increased F-actin polymerization. Thus, the formation of BCL2 and gelsolin complexes (which possibly contain other proteins) appears to play a critical role in the regulation of cell adhesion and migration. Given the established correlation of cell motility with cancer metastasis, this result may explain why the expression of BCL2 in some tumor cell types reduces the potential for metastasis and is associated with improved patient prognosis.

Keywords: BCL2, gelsolin, motility, actin polymerization

Cell Research (2010) 20:458-469. doi: 10.1038/cr.2010.21; published online 9 February 2010

\section{Introduction}

$B C L 2$ was initially classified as a proto-oncogene because of its anti-apoptotic function [1] and the observation that BCL2 expression resulted in weak tumorigenesis in a transgenic mouse model [2]. BCL2 expression in endothelial cells was reported to enhance tumor metastasis [3]. Recently, it has been shown that BCL2 expression may, in some cases, be associated with improved

Correspondence: John E French ${ }^{\mathrm{a}}$, Hengning $\mathrm{Ke}^{\mathrm{b}}$

${ }^{\mathrm{a}} \mathrm{Tel}:+1$ 919-541-2569

E-mail: french@niehs.nih.gov

${ }^{\text {b}}$ Tel: +1 919-684-8037

E-mail: hk71@notes.duke.edu

Abbreviations: BCL2 (human gene); Bcl2 (mouse gene); BCL2 (human and mouse protein)

Received 25 March 2009; revised 18 August 2009; accepted 20 November 2009; published online 9 February 2010 prognosis of patients diagnosed with non-small-cell lung cancer [4], renal cell carcinoma [5], colorectal cancer [6], and melanoma [7]. BCL2 expression was not only correlated with an improved prognosis but also with a reduced capacity for distant colonization of breast cancer cells [8]. Furthermore, the expression level of BCL2 within differentiated cells changes markedly during tissue development and tumorigenesis [9]. However, the physiological role of the BCL2 expression in cell motility has not been well characterized.

Cell adhesion and motility are fundamental functions of both normal cells and metastatic tumor cells, involving both transmembrane adhesion receptors and intracellular signaling molecules [10-14]. These signaling molecules modulate a wide range of intracellular events such as regulation of actin polymerization, which is necessary for cells to change shape, form lamellipodia, and migrate [15-19]. The cycle of actin polymerization and depo- 
lymerization is regulated by a number of actin-binding proteins, one of which is gelsolin. A normal functioning of these actin-binding proteins is required for controlling the rate of actin-based cell migration.

The actin-capping and actin-severing protein, gelsolin, is thought to be critical to the regulation of focal adhe- sion, podosome, invadopodium, and other critical functions that govern normal processes, such as wound healing and also disease processes, such as tumor progression [20-22]. The critical functions of gelsolin include maintaining G-actin concentrations by its F-actin-severing activity and capping the fast growing ends of polymerized
A

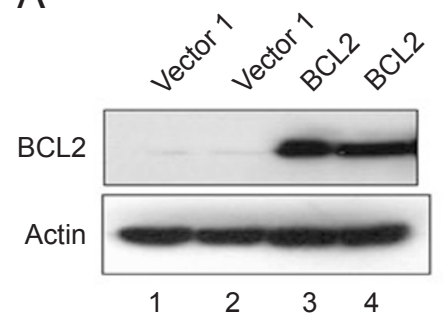

C

MCF7-vector
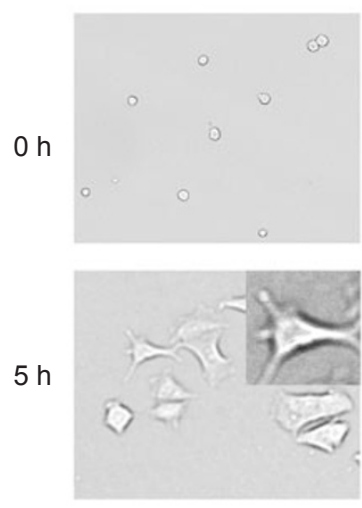

$E$

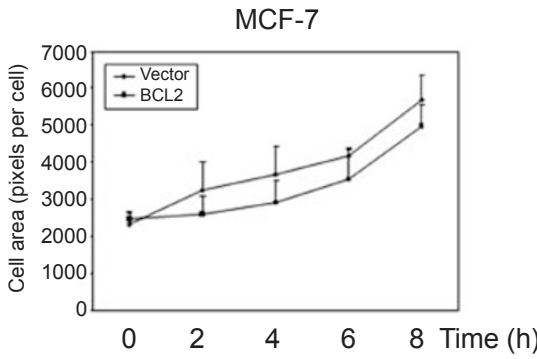

B

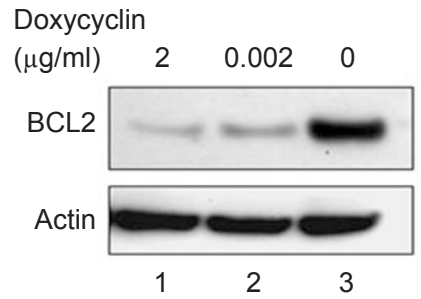

D

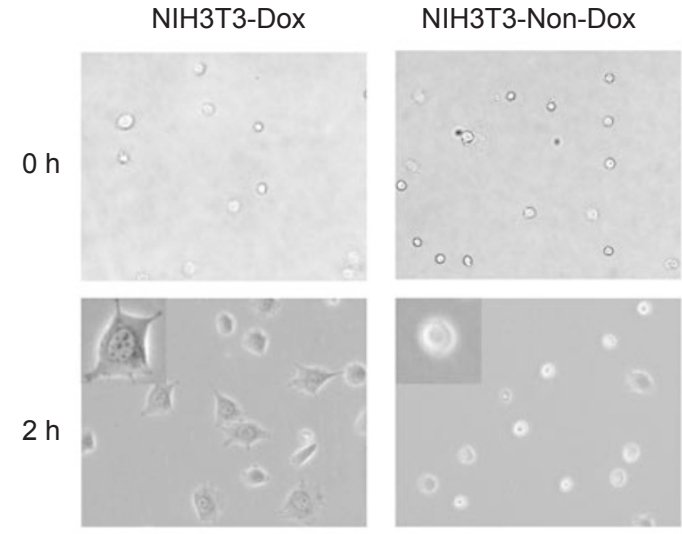

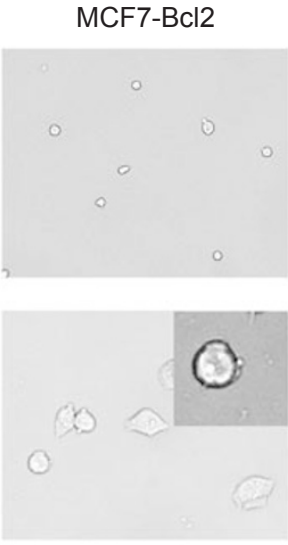

$\mathrm{NIH} 3 \mathrm{~T} 3$

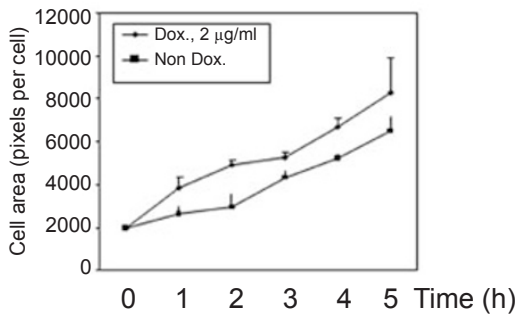

Figure 1 BCL2 expression in human MCF-7 cells and mouse NIH3T3 cells affect cell spreading. (A) Immunoblot analysis of two independent MCF-7-BCL2 clones (lanes 3 and 4) showed higher expression of BCL2 than two MCF-7-vector clones (lanes 1 and 2). (B) Doxycycline-inducible NIH3T3-Bc/2 cells expressed varying levels of BCL2 depending on the concentration of doxycycline. Cells were cultured for 3 days in the presence of $2 \mu \mathrm{g} / \mathrm{ml}$ (lane 1), $0.002 \mu \mathrm{g} / \mathrm{ml}$ (lane 2) doxycycline or without doxycycline (lane 3). BCL2 expression was analyzed by immunoblot (C, D). Phase microscopy images indicated that BCL2 expression in MCF-7 cells (C) and NIH3T3 cells (D) inhibited cell spreading. The cells were cultured with or without doxycycline for 3 days. Cells were harvested and replated on collagen-coated plates, and the spreading was observed for 5-8 hours. In both MCF-7 and NIH3T3 cells, BCL2 expression appeared to result in the presence of more unspread cells. (E) Quantitative analysis of the cell area during spreading confirmed that BCL2 expression significantly (MCF-7 cells, at $0 \mathrm{~h}$ time point, $P$ $=0.53$; at 2, 4, 6, $8 \mathrm{~h}$ time point $P<0.01$. NIH3T3 cells, at $0 \mathrm{~h}$ time point, $P=0.2$; at $1,2,3,4,5 \mathrm{~h}$ time point, $P<0.01)$ inhibited spreading of both MCF-7 cells (left panel) and NIH3T3 cells (right panel). The results were expressed as averaged pixels per cell. 
A

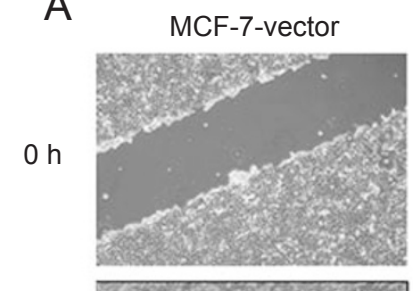

$24 \mathrm{~h}$

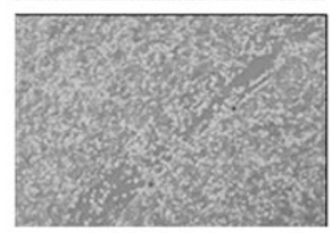

C
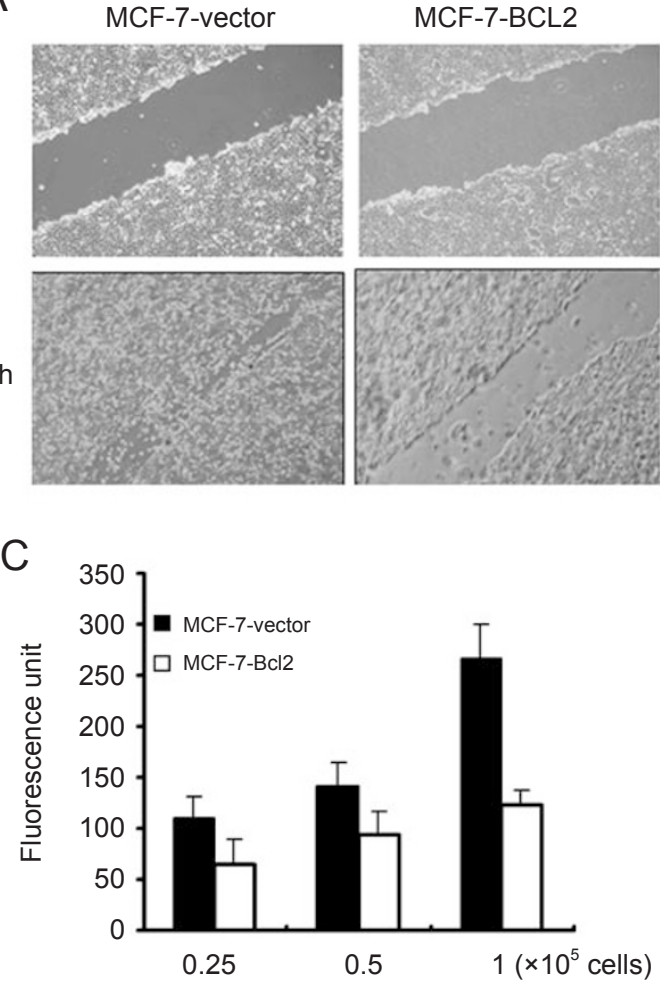

B
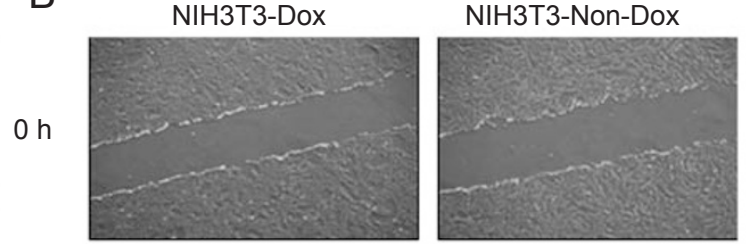

$16 \mathrm{~h}$
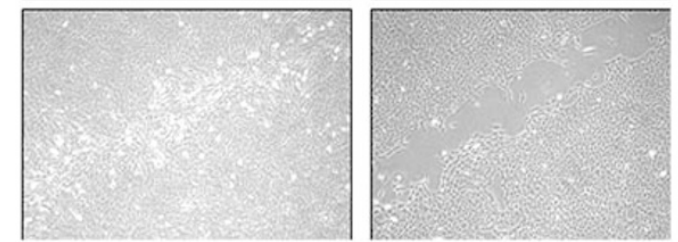

$\mathrm{D}$

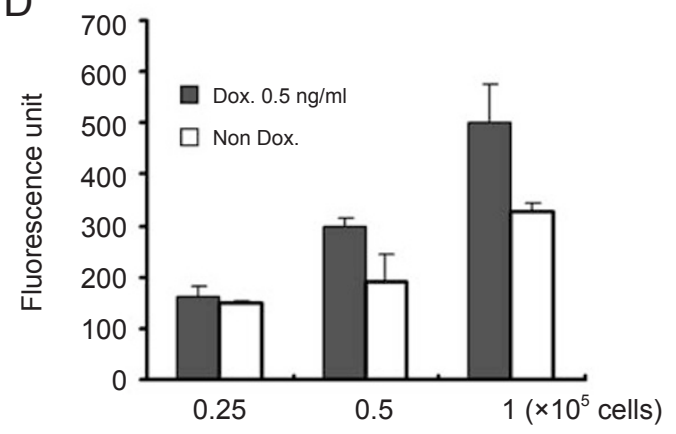

Figure 2 BCL2 expression inhibited cell motility. (A) After $24 \mathrm{~h}$ of incubation, there were more cells that moved into and filled the gap by MCF-7-vector cells (left) compared to MCF-7-BCL2 cells (right). (B). After $16 \mathrm{~h}$ of incubation, there were more cells that moved into and filled the gap by NIH3T3 cells that do not overexpress BCL2 (Dox., left) compared to NIH3T3 that overexpressed BCL2 (non-Dox., right). (C) Quantitative analysis of cell motility by transwell cell migration assay is described in the "Materials and Methods" section. Approximately $50 \%$ fewer cells migrated into the chamber containing $10 \%$ FBS in MCF-7 cells that overexpressed BCL2 (white) compared to MCF-7 that expressed normal levels of BCL2 (black) (all groups, $P<0.01$ ). (D) NIH3T3 cells that overexpressed BCL2 migrated significantly less (white bars) compared to those with native BCL2 expression (black bars), $\left(0.25 \times 10^{5}\right.$ cells, $P=0.7 ; 0.5 \times 10^{5}$ cells, $P<0.05 ; 1 \times 10^{5}$ cells, $\left.P<0.05\right)$. The results were the average value of three experiments.

actin to regulate the growth and average length of F-actin filaments [20, 23]. Membrane ruffling was decreased in fibroblasts without gelsolin expression [24], which demonstrated the importance of gelsolin function in motility. On the other hand, expression of gelsolin enhances cytoskeleton reorganization and cell motility $[25,26]$.

To understand better the role of BCL2 in the mechanism of cell adhesion and migration, we used a doxycycline-controlled system to express murine BCL2 in cultured NIH3T3 cells and expressed human BCL2 in cultured MCF-7 human mammary adenocarcinoma cells. We found that BCL2 expression inhibited cell adhesion, spreading, and motility. We also found the formation of a novel complex that contains BCL2, actin, and gelsolin that may play a role in the regulation of actin polymerization and de-polymerization. To our knowledge, this is the first report showing that BCL2 can function as a regulatory molecule for actin polymerization in a manner that regulates cell adhesion, spreading, and motility.

\section{Results}

\section{Expression of BCL2 inhibited cell spreading}

To characterize the consequences of BCL2 expression on cell adhesion, spreading, and motility, human BCL2 expression and empty vector (control) cell lines were established by transfection of pcDNA3-BCL2 or pcDNA3 into cultured MCF-7 cells. The derivative clones that were stably transfected with the $B C L 2$ gene were identified by western blot analysis (Figure 1A). Another model used a doxycycline-responsive NIH3T3 cell line transfected with the mouse $B c l 2$ gene. Different levels of BCL2 expression could be obtained by varying the dosage of doxycyline (Figure 1B).

We monitored cells for both shape and spreading after incubation on collagen type-IV substrates. Cells with 
BCL2 expression tend to spread isotropically, while vector-transfected cells spread anisotropically, as shown in Figure 1C-1E, and the cell area increased in a timedependent manner. Both the rate and the extent of cell spreading were significantly lower $(P<0.01$ is indicated at all time points except the $0 \mathrm{~h}$ point) in BCL2-over- expressing NIH3T3 cells and human MCF-7 cells. This result suggests that overexpressed BCL2 may inhibit cell spreading.

\section{Expression of BCL2 inhibited cell migration}

Because of the striking effects of BCL2 expression

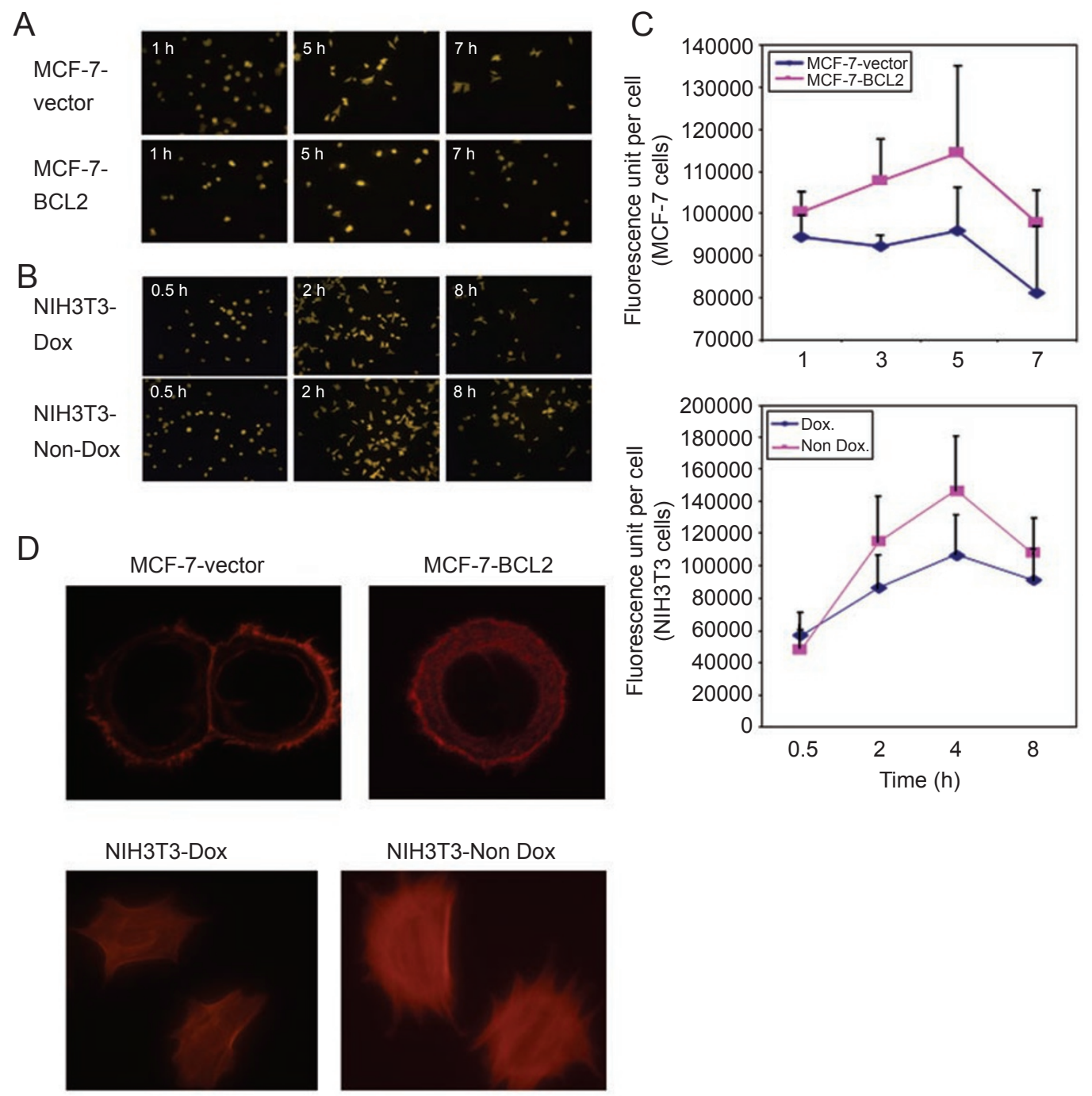

Figure 3 BCL2 expression enhanced F-actin polymerization during cell spreading in vivo. Cultured cells were fixed and and then stained with $165 \mathrm{nM}$ rhodamine phalloidin in PBS for 20 min. Five images were taken and the intensity of fluorescence was analyzed using a Zeiss LSM Imager. At least 500 randomly selected cells per data point were measureded to determine an average fluorescence value. The experiments were repeated twice with similar results. (A) In vivo F-actin staining of MCF7 cells showed that F-actin increased during cell spreading, peaked at $5 \mathrm{~h}$, and was reduced thereafter. (B) In vivo F-actin staining of NIH3T3 cells showed that F-actin increased during cell spreading, peaked at $4 \mathrm{~h}$, and was reduced thereafter. (C) Quantitative fluorescence analysis showed that the average value per cell was higher in MCF-7 cells that overexpressed BCL2 (upper panel, at 1, 3, 5, $7 \mathrm{~h}$ time points, $P<0.01$ ) and NIH3T3 cells (lower panel, at $0.5 \mathrm{~h}$ time point, $P=0.48$; at 2, 4, $8 \mathrm{~h}$ time points, $P<0.01$ ). These results indicated that BCL2 expression significantly increased F-actin polymerization. (D) Representative F-actin staining of individual cell indicated that MCF-7-BCL2 cells have more extensive F-actin staining in the cytoplasma than do MCF-7-vector cells (upper panels). Representative F-actin staining of individual cells indicated that NIH3T3-non-Dox (right lower panel) have more extensive F-actin staining in the cytoplasm than do NIH3T3-Dox cells (left lower panel). 
on cell attachment and spreading, we examined the effects of BCL2 expression on cell migration. Migration of both MCF-7 and NIH3T3 cells was first analyzed using a scratch wound closure assay [27]. MCF-7 cells overexpressing human BCL2 demonstrated little migration into the wounded area. In contrast, MCF-7 cells transfected with the empty vector migrated readily across the wound (Figure 2A). Similarly, NIH3T3 cells cultured without doxycycline, which overexpressed mouse BCL2, appeared to migrate more slowly into the wounded area than did those cultured in the presence of doxycycline, which expressed lower levels of Bcl2 and appeared to migrate faster, completely filling the wound area within $16 \mathrm{~h}$ (Figure 2B). Similarly, expression of mouse BCL2 in B6 melanoma cells caused decreased cell migration (see Supplementary information, Figure S1). Transwell cell migration was also quantified using DMEM containing $10 \%$ fetal bovine serum (FBS) as the chemoattractant [28]. Expression of BCL2 in MCF-7 cells inhibited cell motility by over $50 \%(P<0.01)$ (Figure $2 \mathrm{C}$ ) and by $\sim 30 \%$ in NIH3T3 cells $(P<0.05)$ (Figure 2D). These results suggest that, in both murine and human cells, lower levels of expression of BCL2 may be optimal for cell migration, while higher levels of expression of BCL2 inhibited cell motility.

BCL2 expression enhanced $F$-actin polymerization during cell spreading

Previously, it has been shown that F-actin polymerization increases for the first several hours during cell spreading and then decreases over $24 \mathrm{~h}$ later [29]. We examined the effect of BCL2 expression on F-actin polymerization in vivo during cell spreading. The cells were fixed and stained with rhodamine phalloidin, and the Factin amount was quantified by measuring the total fluorescence intensity of 500 cells at different time points. As judged by phalloidin staining, the amount of F-actin in MCF-7-vector cells increased during the first several hours after seeding and peaked after $5 \mathrm{~h}$ (Figure 3A). BCL2-overexpressing MCF-7 and NIH3T3 cells both had higher fluorescence intensities, suggesting increased F-actin content (Figure 3B). Quantitative analysis of fluorescence indicated that the average fluorescence value per cell is significantly higher in MCF-7 cells $(P<0.01$ in each of the time points) (Figure 3C, upper panel) and in NIH3T3 cells $(P<0.01$ in each of the time points, except the initial time point) (Figure 3C, lower panel) that are overexpressing BCL2. Interestingly, the representative F-actin staining of cells showed obviously more Factin in the cytoplasm of MCF-7-BCL2 cells or NIH3T3non-Dox cells compared with MCF-7-vector cells or NIH3T3-Dox cells (Figure 3D). These results suggest that increasing BCL2 expression results in a concommitant increase of F-actin within cells.

Loss of Bcl2 results in increased cell migration and decreased actin polymerization rate

To further study the effect of the level of expression of BCL2 on cell motility, we compared the migration of Bcl2-null MEFs with wild-type MEFs. As shown in Figure 4A, Bcl2 -/- MEFs had an increased migratory phenotype - these cells completely filled the gap after a 48 -h incubation. In contrast, $B c l 2+/+$ cells did not close the gap after $48 \mathrm{~h}$. Furthermore, cell lysates from $\mathrm{Bcl} 2$ null MEFs significantly inhibited actin polymerization in the in vitro actin polymerization assay. In contrast, cell

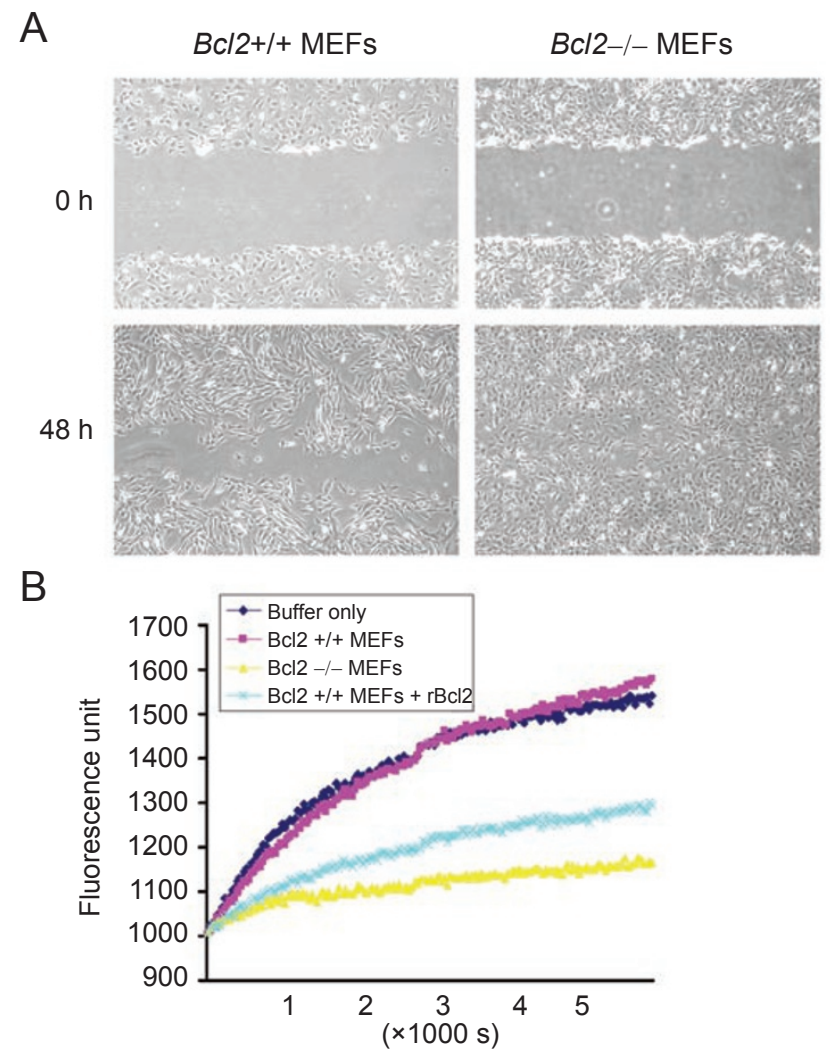

Figure 4 Loss of $\mathrm{Bc} / 2$ results in increased cell migration and lower actin polymerization rate. (A) Bc/2 -/- MEFs (right) displayed higher motility, completely filling the "wound" after 48 h. In contrast, Bcl $2+/+$ cells (left) did not completely close the "wound" after $48 \mathrm{~h}$ of incubation. (B) Using an in vitro actin polymerization assay, cell lysates from Bc/2-null MEFs (yellow) markedly inhibited actin polymerization, while the same amount of cell lysate from $\mathrm{Bcl} 2+/+$ MEFs had little effect on actin polymerization (red) compared to the control of buffer only (dark blue). Addition of $0.5 \mu \mathrm{g}$ recombinant human BCL2 to the lysate of Bc/2 -/- MEFs partially restored the level of actin polymerization in the in vitro assay (light blue). 
lysates from $\mathrm{Bcl} 2+/+$ MEFs had little effect on actin polymerization compared with buffer-only controls. Addition of $0.5 \mu \mathrm{g}$ of recombinant human BCL2 to the lysate of $\mathrm{Bcl} 2-/-$ MEFs partially reversed the inhibition of actin polymerization caused by $B c l 2-/-$ cell lysates in the in vitro assay (Figure 4B). Interestingly, addition of the BCL2 antibody partially decreased actin polymerization (see Supplementary information, Figure S2), suggesting that the presence of BCL2 itself may be important for its effect on F-actin polymerization. These results are consistent with a mechanism by which the expression of BCL2 may enhance actin polymerization and negatively regulate cell motility.

\section{BCL2 can bind actin and complex with gelsolin}

To begin to characterize the mechanism of BCL2 modulation of F-actin polymerization, we used a proteomic approach to identify proteins that could bind BCL2 from NIH3T3 cells that overexpressed BCL2. In addition to actin, a 97-kDa protein was found to complex with BCL2 and was identified as murine gelsolin by mass spectrometry (Table 1). To further investigate whether BCL2 bind actin directly and how BCL2 and gelsolin complex together, we first used recombinant BCL2 and actin protein to perform an immunoprecipitation assay. Human recombinant BCL2 was found to bind to actin directly by immunoprecipitation analysis (Figure 5A). Then, immunoprecipitation and immunoblotting analyses indicated that both in the presence and absence of latrunculin B, gelsolin could be coprecipitated with the BCL2 antibody in MCF-7-BCL2 cells. BCL2 can also be pre-

Table 1 Proteins precipitated by anti-BCL2 antibody

\begin{tabular}{lll}
\hline \multicolumn{1}{c}{ Protein } & Accession & \#MW \\
\hline Ribosome protein S19 & gi12963511 & 16076 \\
Ribosome protein S10 & gi127170 & 19883 \\
Ribosome protein 3A & gi3914914 & 29481 \\
tropomyosin & gi1351289 & 32675 \\
actin & gi1703121 & 41809 \\
translation elongation factor 1 $\alpha 1$ & gi15277711 & 42997 \\
vimentin & gi2078001 & 51533 \\
tubulin $\alpha 1$ & gi6755901 & 50104 \\
protein disulfide-isomerase precursor & gi68462 & 57023 \\
glucose-regulated protein precursor (GRP 78) & gi121570 & 72334 \\
nucleolin & gi7106377 & 76677 \\
gelsolin & gi6754078 & 80827 \\
procollagen, type I, $\alpha 2$ & gi6680980 & 129524 \\
collagen $\alpha 1$ & gi2506350 & 137859 \\
myosin & gi6981236 & 226197 \\
\hline
\end{tabular}

cipitated with the gelsolin antibody by coimmunoprecipitation and immunoblotting analyses (Figure 5B). Finally, confocal images indicated that BCL2 and gelsolin colocalize in the membrane and cytoplasm of MCF7-BCL2 cells, respectively (Figure $5 \mathrm{C}$ ). These results suggest that BCL2 and gelsolin can form a complex possibly through actin in both mouse and human cell lines.

We also examined whether human BCL2 could bind to human gelsolin directly in vitro. A GST pull-down assay showed that BCL2 could bind to BAD but not to the GST-gelsolin fusion protein (Figure 5D). This result suggests that BCL2 may not bind directly to gelsolin in vivo.

BCL2 expression inhibited the actin-severing activity of gelsolin while enhancing actin polymerization in vitro

Inhibition of the gelsolin-severing activity has been shown to result in decreased cell migration; conversely, increasing intracellular gelsolin resulted in increased cell migration [26]. We therefore tested the hypothesis that BCL2 inhibition of cell motility could be mediated by gelsolin. Total gelsolin expression appeared to be unaffected by the expression of BCL2 in both NIH3T3 and MCF-7 cells, although the gelsolin expression in NIH3T3 cells was lower than that of MCF-7 cells (Figure $6 \mathrm{~A})$. When tested in an in vitro, fluorescence-based assay for F-actin-severing activity [30], the fluorescence intensity of the reaction containing lysates from MCF7 cells transfected with only empty vector decreased within minutes. In contrast, no fluorescence decay was observed in the reaction with lysates containing the same amount of total protein from cells that overexpress BCL2 (Figure 6B). This result suggests that lysates from cells that overexpress BCL2 show decreased gelsolin-severing activity compared to cell lysates from MCF7-vector cells containing normal levels of expression of BCL2.

To further address the role of BCL2 expression in the process of G-actin polymerization to F-actin, we assayed the polymerization of purified G-actin in the presence of cell lysates from control cells and those overexpressing BCL2. As shown in Figure 6C, the lysate from control MCF-7-vector cells caused only a slight increase in fluorescence, suggesting the presence of actin-severing activity, while the lysate from MCF-7 cells overexpressing $\mathrm{BCL} 2$ restored the $\mathrm{F}$-actin polymerization rate to the level of that observed with the normal buffer alone. Similarly, we observed that the lysate from NIH3T3 cells overexpressing BCL2 had an activating effect on F-actin polymerization (Figure 6D). While the actin polymerization agent, jasplakinolide, increased the actin polymerization rate, the actin depolymerization agent, latrunculin $\mathrm{B}$, significantly decreased the actin polymerization rate. These results confirm the finding that a higher level of 
A

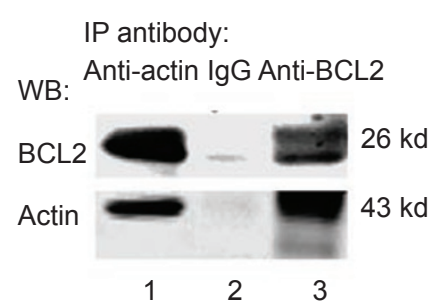

B

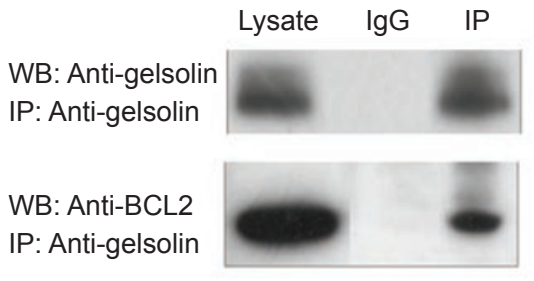

$97 \mathrm{kd}$

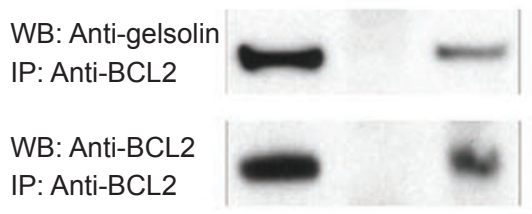

Without latrunculin B
$97 \mathrm{kd}$

$26 \mathrm{kd}$
IP antibody:

Anti-BCL2 IgG Anti-gelsolin

WB:

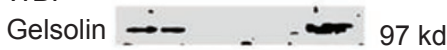

$\mathrm{BCL} 2$

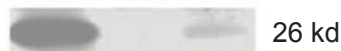

In the presence of latrunculin B
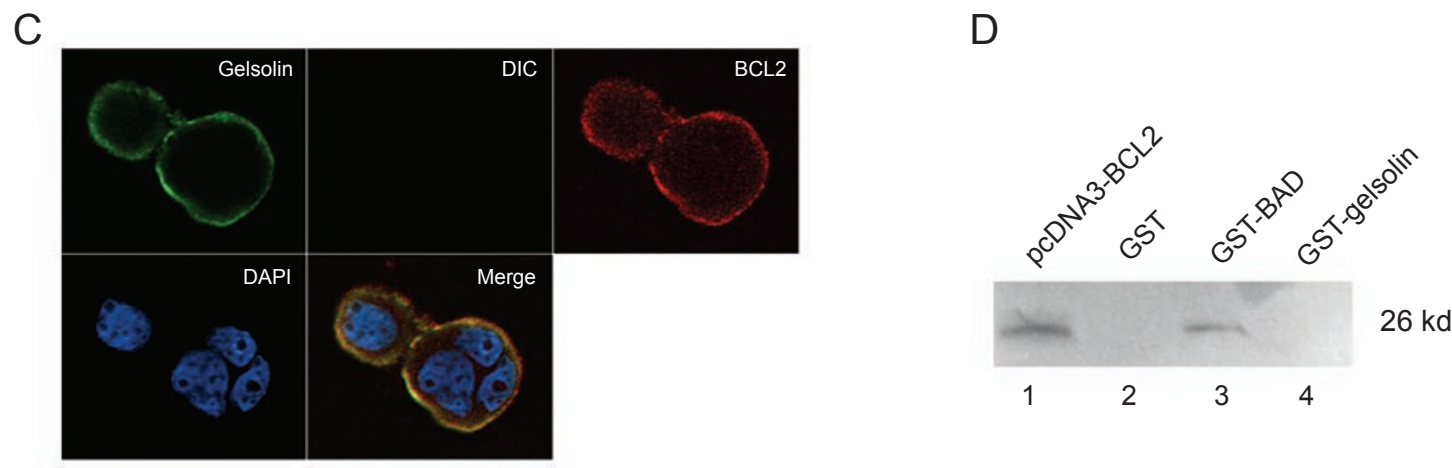

Figure 5 BCL2 binds actin and complexes with gelsolin. (A) Recombinant human BCL2 was mixed with actin, and immunoprecipitation was carried out as described in the "Materials and Methods" section. Both proteins were precipitated by either rabbit anti-BCL2 or goat anti-actin antibody as shown in lanes 1 and 3 . The antibodies used in western blot were either mouse anti-human BCL2 or rabbit anit-actin, respectively. The rabbit lgG control in lane 2 shows that nothing is immunoprecipitated in the absence of specific antibodies. (B) Gelsolin and BCL2 were coimmunoprecipitated from lysates of MCF-7 that overexpress BCL2 (MCF-7-BCL2 cells) with either goat anti-gelsolin or mouse anti-human BCL2 (BD Biosciences). The antibodies used in western blot were either mouse anti-gelsolin or mouse anit-human BCL2 (BD Biosciences), respectively. The experiment in the left pannel was performed in the absence of latrunculin B. The complex containing both Bcl2 and gelsolin was precipitated using either anti-gelsolin (top) or anti-BCL2 (bottom) antibody. In contrast, mouse IgG can precipitate neither gelsolin nor BCL2 (middle lanes of both top and bottom). The experiment in the right panel was performed in the presence of $0.5 \mu \mathrm{g} / \mathrm{ml}$ latrunculin B with similar results. (C) Confocal microscopy showed the colocalization of gelsolin with BCL2 in MCF7-BCL2 cells. The merged image indicated that both gelsolin and BCL2 were located in the cell membrane and cytoplasm. These experiments were repeated twice with similar results. (D) Using the GST pull-down assays, BCL2 was found to be bound to BAD (lane 3), but did not bind directly to gelsolin in vitro (lane 4). Lane 1 showed the in vitro translated human BCL2 protein. Lane 2 showed a GST-negative control. The pull-down proteins were separated by electrophoresis and visualized with Typhoon 8600 Variable Mode Imager.

BCL2 expression enhances F-actin polymerization.

\section{Discussion}

We have shown that increased expression of BCL2, a multifunctional protein, inhibited cell adhesion, spreading, and migration. The mechanistic basis for these effects appears to involve enhanced actin polymerization and reduced gelsolin-severing activity, which is associ- ated with the formation of a complex that contains both gelsolin and BCL2. These results suggest that BCL2 may function as a noval cytoplasmic factor that regulates cell motility.

The area of the cell adhering to the substrate during spreading is thought to be related to the total magnitude of force exerted by the cell $[29,31]$. Force is again generated when actin monomers polymerize into actin filaments in the plasma membrane. The reduction of the 
spreading of cells that overexpress BCL2 suggests that there may be abnormal actin polymerization close to the plasma membrane. We show here that actin polymerization and depolymerization are directly modulated by the level of BCL2 expression and are linked to both cell spreading and cell motility. Consistent with this observation, BCL2 overexpression in MCF-7 cells inhibits the cell-cell adhesion caused by $12 \mathrm{G} 10$ antibody, resembling the effects of an actin polymerization agent (see Supplementary information, Figure S3). Previously published studies showed that the expression of BCL2 results in an increase of a simple attachment of osteoblasts to Primaria substrates [32], and knocking out BCL2 results in a decreased attachment of ureteric bud cells to vitronectin and fibronectin [33]. These studies use a different end point (simple cell attachment) than does our study (cell spreading), which could possibly explain the different

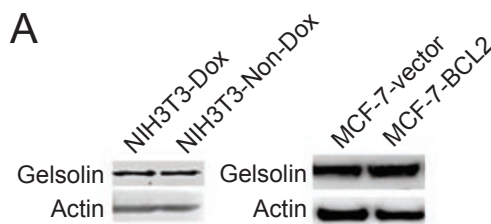

B
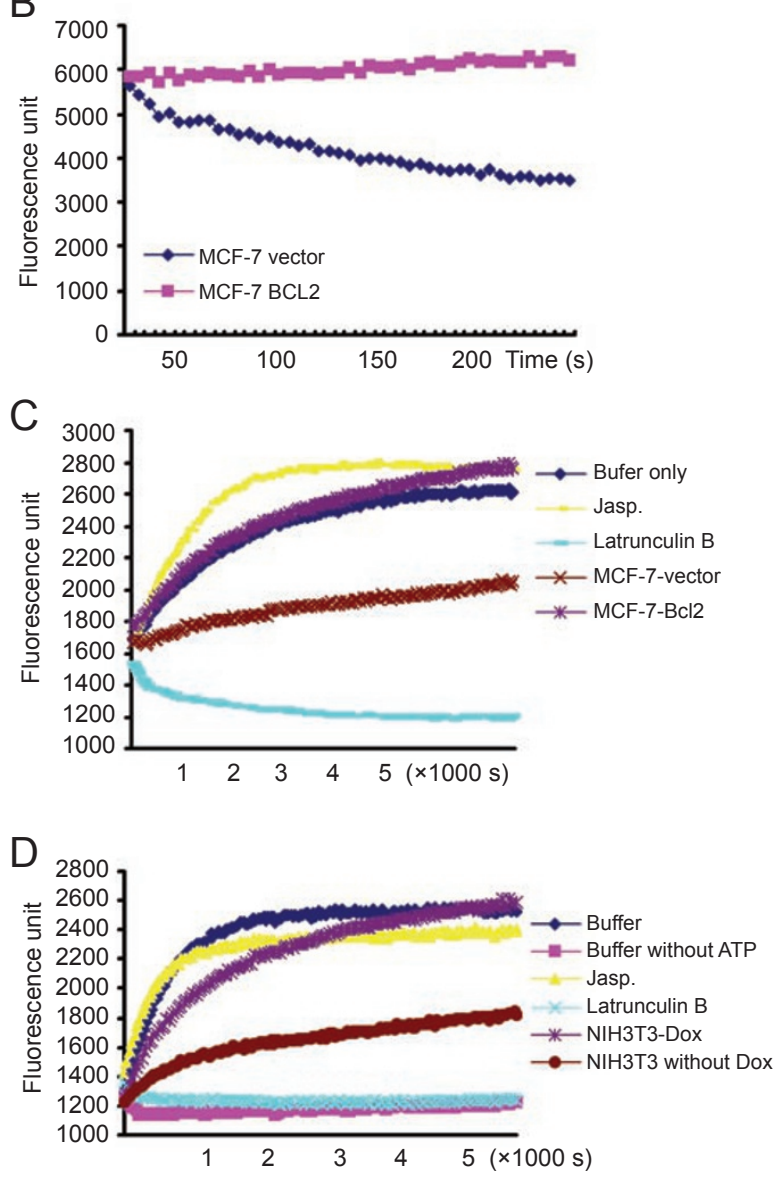

conclusions.

Cell migration involves a multistep process that requires formation of new adhesions at the leading edge and the breaking of adhesions at the trailing edge [16, 34-36], a process that is mediated by integrin receptors on the cell surface $[10,37-42]$. Cell migration also requires cytoskeltal remodeling, a process that is mediated by a number of cytoplalsmic proteins one of which is gelsolin $[34,43,44]$. It is thought that it is the amount of free gelsolin level relative to its severing activity that is critical, rather than simply the total amount of gelsolin [26]. Our results are consistent with these findings and further suggest that gelsolin can form a complex with other proteins that may reduce its severing activity but enhance the nucleation function. The nucleating activity of gelsolin at the plasma membrane predominates in lamellipodia, membrane ruffles, and adhesion foci [45]. Gelsolin associates with membrane through polyphosphoinositides and non-polyphosphoinositides

Figure 6 BCL2 expression downregulated gelsolin-severing activity and enhanced actin polymerization in vitro. (A) Gelsolin expression level was not changed by BCL2 expression in either NIH3T3 cells (left panel) or MCF-7 cells (right panel). (B) Rhodamine phalloidin-labeled $\mathrm{F}$-actin was incubated with dialyzed protein lysate from vector-transfected (red) or BCL2-overexpressing (blue) MCF-7 cells in the presence of calcium. Fluorescence recording indicated that the gelsolin-severing activity was inhibited in cell lysates from MCF-7 cells that overexpressed $\mathrm{BCL}$ 2. The fluorescence decayed within minutes in control MCF7-vector cells (blue), indicating a normal severing activity causing the loss of F-actin. These experiments were repeated three times and yielded similar results each time. (C) The actin polymerization assay was peformed with controls of buffer only, buffer without ATP, buffer in the presence of either $1 \mu \mathrm{M}$ jasplakinolide, an actin polymerization agent, or $0.5 \mu \mathrm{g} / \mathrm{ml}$ latrunculin $\mathrm{B}$, an actin depolymerization agent. Pyrene actin $(2.3 \mu \mathrm{M})$ was incubated with $10 \mu \mathrm{g}$ of dialyzed cell lysates in polymerization buffer. Actin polymerization was dramatically inhibited by latrunculin (light blue). The actin polymerization process was normal or increased when incubated with buffer (dark blue) or jasplakinolide (yellow). Lysates from MCF-7-vector cells consisting of $10 \mu \mathrm{g}$ total protein inhibited actin polymerization (brown). Lysates (10 $\mu \mathrm{g}$ total protein) from MCF-7 cells that overexpressed BCL2 enhanced actin polymerization (purple). (D) Compared to bufferonly control conditions (dark blue), lysates containing $10 \mu \mathrm{g}$ total protein from NIH3T3 cells (doxycycline added) inhibited actin polymerization (brown), while $10 \mu$ g lysates from NIH3T3 cells (without doxycycline) that overexpressed $\mathrm{Bcl} 2$ enhanced actin polymerization (purple). Latrunculin B completely inhibited F-actin polymerization (light blue) down to the level of negative control conditions from which ATP was deleted (magenta). Jasplakinolide also activated F-actin polymerization (yellow). This experiment was repeated three times with each of the above cell lines and yielded similar results. 
lipids [46, 47]. The binding of these molecules can dissociate gelsolin from the capped actin and increase the amount of F-actin [48]. Our finding that cytosol from MCF-7 cells that overexpress BCL2 has decreased the gelsolin-severing activity and higher actin polymerization rates, as judged by in vitro assays, suggests that the rate and amount of actin monomers in the region behind lamellipodium recycling to the cell front (treadmilling) to extend the lamelliopodium protrusion may reduce. Interpretation of results of the gelsolin-severing assay requires careful consideration since other severing proteins might affect the actin depolymerization. We propose that gelsolin may be functionally more important in mediating this BCL2 function. Finally, our results are consistent with those from previously published studies showing that BCL2-null cells have a greater motility [33, 49]. We hypothesize that rapid F-actin assembly and disassembly, which are required to maintain normal cell elasticity, cell adhesion, and cell motility, may be crippled in cells overexpressing BCL2 possibly because of the downregulation of gelsolin-severing activity.

We have also described a novel gelsolin-BCL2 complex in cells that overexpress BCL2, suggesting that signaling complexes may exist to directly inhibit actin depolymerization and thus affect cytoskeleton remodeling. We did not observe a striking change of Rac-GTP expression induced by BCL2 expression (data not shown), indicating that there may be an alternate, Rac-GTPindependent, pathway by which BCL2 inhibits gelsolin function and actin depolymerization. It is likely that both BCL2 and gelsolin bind actin and therefore complex together. The scaffolding adaptor protein, paxillin, was also detected in BCL2 antibody-precipitated complex (data not shown), confirming the findings by others that paxillin can bind both BCL2 and gelsolin $[50,51]$ to form a complex that can regulate physiological processes that require cell migration $[50,52]$. The mechanism by which BCL2 complexes with gelsolin has not been fully investigated, but will be important to determine. We propose that BCL2 complexes with actin, paxillin, gelsolin, and other integrin-associated proteins, and negatively regulates motility-related pathways.

The finding that BCL2 expression can inhibit the motility of certain cell lines suggests that there may be a reduced potential of these cells to metastasize and may help explain why there is a higher survival rate in patients with certain tumor types that overexpress BCL2 [53]. This conclusion is, however, inconsistent with other reports that BCL2 may enhance tumor growth and metastasis $[54,55]$. The critical parameter may, in fact, be the overall balance between the anti-apoptotic and the anti-migratory activities of BCL2 that determines whether BCL2 expression is associated with increased or decreased survival in cancer patients.

The BCL2-gelsolin regulation of F-acting depolymerization may have a broader significance for cell division and apoptosis since both processes require cytoskeleton reorganization. We propose that downregulation of the gelsolin-severing activity and decreased disassembly of F-actin induced by BCL2 expression may be a novel mechanism by which apoptosis can be inhibited. For example, BCL2 can attenuate cadmium-induced apoptosis and necrosis [56, 57]. Cadmium also causes association of gelsolin with actins and subsequent loss of F-actin during apoptosis [30]. Therefore, we speculate that BCL2 may also inhibit apoptosis by affecting the gelsolinsevering activity.

In conclusion, we have demonstrated that expression of BCL2 in two distinct cell lines inhibited cell adhesion and migration via a mechanism involving inhibited gelsolin-severing activity and enhanced actin polymerization. The finding that BCL2 may regulate cell motility and adhesion should also be very useful for the investigation of the role of BCL2 in tumor metastasis, tissue and organ development, and homing of stem cells to damaged and regenerating tissues.

\section{Materials and Methods}

\section{Plasmids and cell culture}

The pcDNA3-BCL2 plasmid was obtained by inserting a Not I cut fragment from pCMV-XL5- $h B C L 2$ containing human $B C L 2$ cDNA (Accession: NM 00063, OriGene Technologies) into the NotI site of pcDNA3. The pcDNA3-BCL2 or pcDNA3 empty vector was transfected separately into the MCF-7 cells (ATCC, Masassas, VA, USA) with lipofectamine (Invitrogen). The cells were cultured in 10\% DMEM containing 10\% FBS (Clontech, Mountain View, CA, USA) for $24 \mathrm{~h}$ after transfection, then replated into 150 $\mathrm{mm}$ culture dishes in culture media supplemented with G418 (750 $\mu \mathrm{g} / \mathrm{ml}$ ), and cultured for 14 days of growth. Visible clones were isolated and transferred to individual dishes. The derivative clones with high expression of the BCL2 protein were obtained and amplified for assays. Fragments of NheI and $\mathrm{XbaI}$ (blunt) of murine $B c l 2$ from pUSEamp- $B c l 2$ (Upstate) were subcloned into the NheI and NotI (blunt) sites of pTRE2hyg (Clontech). The resulting plasmid was transfected into the NIH3T3 Tet-off cell line (Clontech). Doxycycline-responsive cell clones were selected by culturing the transfected cells in the presence of hygromycin $(300 \mu \mathrm{g} / \mathrm{ml})$ and identified by western blot analysis. The cells were maintained in DMEM containing $10 \%$ FBS plus hygromycin $(200 \mu \mathrm{g} / \mathrm{ml})$, with or without Doxycycline (Dox., $2 \mu \mathrm{g} / \mathrm{ml}$ ). The Bcl2 wild-type MEFs and Bcl2-null MEFs were generous gifts from Dr Christine M Sorenson (University of Wisconsin, Madison). MEF cells were grown in DMEM containing $10 \%$ fetal calf serum (FCS) plus 5.3 $\mathrm{ng} / \mathrm{ml}$ of $\gamma$-interferon (R\&D Inc.), $10^{-5} \mathrm{M} \beta$-mercaptoethanol.

\section{Cell spreading}

For cell spreading assays, cells were plated onto collagen type- 
IV coated wells at 5500 cells per $\mathrm{cm}^{2}$ in DMEM. Five images were taken from random locations at 1.5-2 $\mathrm{h}$ intervals using phase microscopy (Olympus, IX70, Tokyo, Japan). The cell surface area was determined and calculated automatically by image analysis using a FluoChemTM IS-8900 (Alpha Innotech). Each data point is the average of five images \pm SEM.

\section{Scratch wound assay and transwell cell migration assay}

The migration of cells was assessed using a scratch wound assay [58]. Approximately 5-10 $\times 10^{5}$ cells were grown on $35-\mathrm{mm}$ collagen-coated plates for $24-48 \mathrm{~h}$ to form confluent monolayer of cells. Gaps were created in confluent cell layers using micropipette tips, rinsed with DMEM media. Cells were then cultured with the DMEM media with $1 \%$ FBS (or 2\% FCS for MEFS) to minimize the effect of cell proliferation. The wound closure was monitored by phase-contrast microscopy and photographed over $16 \mathrm{~h}$ for murine or $24 \mathrm{~h}$ for human cells.

Transwell migration assays with serum as the chemoattractant were performed using the QCM 24-Well Fluorimetric Cell Migration Assay kit (Chemicon). Briefly, $500 \mu 1$ DMEM containing 10\% FBS were added to the lower chambers. Cells suspended in $300 \mu \mathrm{l}$ DMEM without FBS were added to the upper compartment of the Transwell unit with $8-\mu \mathrm{m}$ pore size polycarbonate inserts. Cells were allowed to migrate for $16 \mathrm{~h}$ (NIH3T3 cells) or $24 \mathrm{~h}$ (MCF7 cells) at $37{ }^{\circ} \mathrm{C}$ in a humidified atmosphere containing $5 \% \mathrm{CO}_{2}$. The medium with cells from the topside of the inserts was carefully removed by aspiration. The cells from the underside of the inserts were completely dislodged, lysed, and stained by Cyquant GR Dye (Molecular Probes Inc., Eugene, OR, USA). A total volume of 200 $\mu 1$ of cell lysate were then transferred to a 96 -well plate. The plates were read using Spectra MAX Gemini XS (Molecular Devices, Sunnyvale, CA, USA) with a 485/538 nm filter set.

\section{Actin severing and polymerization assays}

The gelsolin-severing activity was measured as described previously [59]. Briefly, $1 \mathrm{mg} / \mathrm{ml}$ purified rabbit muscle actin (Cytoskeleton, Denver, CO, USA) was diluted to $30 \mu \mathrm{g} / \mathrm{ml}$ and resuspended in polymerization buffer $\left(50 \mathrm{mM} \mathrm{KCl}, 2 \mathrm{mM} \mathrm{MgCl}_{2}, 0.5 \mathrm{mM}\right.$ ATP, $2 \mathrm{mM}$ Tris, $\mathrm{pH} 8.0$ ), incubated for $1 \mathrm{~h}$, and sedimented by centrifugation at $100000 \times \mathrm{g}$ for $1 \mathrm{~h}$ to remove the unpolymerized actin. Rhodamine phalloidin ( $1 \mu \mathrm{M}$; Molecular Probes Inc.) was added to actin filaments at a final concentration of $0.4 \mu \mathrm{M}$. The rate of florescence loss at $590 \mathrm{~nm}$ (530-nm excitation wavelength) was measured by fluorometry (FL600, Microplate Fluorescence Reader, BioTEK). Cell lysates from vector-only control cells or MCF-7 cells overexpressing BCL2 was prepared with proteinase inhibitors in lysis buffer containing $50 \mathrm{mM} \mathrm{KCl}, 2 \mathrm{mM} \mathrm{MgCl}_{2}, 0.5$ mM ATP, 2 mM Tris, pH 8.0, 1 mM EGTA, and 1\% Triton X-100. The lysates were dialyzed (Slide-A-Lyzer $10 \mathrm{~K}$, Pierce) at $4{ }^{\circ} \mathrm{C}$ with three changes of buffer containing $50 \mathrm{mM} \mathrm{KCl,} 2 \mathrm{mM} \mathrm{MgCl}_{2}$, $2 \mathrm{mM}$ Tris, $1 \mathrm{mM}$ EGTA, $0.5 \mathrm{mM} \beta$-mercaptoethanol. The volume of cell lysate was adjusted to $400 \mu \mathrm{l}$ using dialysis buffer. The final actin filament concentration in polymerization buffer was $0.4 \mu \mathrm{M}$. The severing assay was started by adding $2 \mathrm{mM} \mathrm{CaCl}_{2}$ and $1 \mathrm{mM}$ EGTA.

Actin polymerization in the presence of cell lysates was measured in a standard pyrene-actin fluorescence assay [30]. Cell lysates were dialyzed as described above. Lysate $(10 \mu \mathrm{l})$ with equal amount of total protein was added to the final reaction vol- ume $(200 \mu \mathrm{l})$. Pyrene actin was diluted to $2.3 \mu \mathrm{M}$ in the general actin buffer ( $5 \mathrm{mM}$ Tris- $\mathrm{HCl} \mathrm{PH} 8.0,0.2 \mathrm{mM} \mathrm{CaCl}_{2}$ ) containing 0.2 $\mathrm{mM}$ ATP and $0.5 \mathrm{mM}$ DTT and stored on ice for $60 \mathrm{~min}$ to depolymerize actin oligomers. The tubes were centrifuged at $14000 \times \mathrm{g}$ force for $30 \mathrm{~min}$ at $4{ }^{\circ} \mathrm{C}$. The supernatants containing pyrene actin were mixed with the lysate, added into the 96-well plate, and held for $3 \mathrm{~min}$. In the control wells, we added either $1 \mu \mathrm{M}$ jasplakinolide, an actin polymerization agent, or $0.5 \mu \mathrm{g} / \mathrm{ml}$ latrunculin $\mathrm{B}$, an actin depolymerization agent. After the addition of $1 / 10$ volume of $10 \times$ of polymerization buffer $(10 \times$ contains $500 \mathrm{mM} \mathrm{KCl}, 10 \mathrm{mM}$ $\mathrm{MgCl}_{2}$ ), the kinetics of pyrene fluorescence upon initiation of actin polymerization was monitored for $2 \mathrm{~h}$ at $405 \mathrm{~nm}$ with an excitation wavelength of $355 \mathrm{~nm}$ with the same Spectra Max Gemini XS.

\section{Confocal immunofluorescence microscopy of protein local- ization}

Approximately $10^{4}$ cells were cultured on $35-\mathrm{mm}$ glass bottom micro dishes (Matek Corp., Ashlan, MA, USA) for 2 days. Cells were fixed with $10 \%$ formalin in PBS for $10 \mathrm{~min}$, followed by permeabilization for $5 \mathrm{~min}$ with ice-cold methanol and $5 \mathrm{~min}$ of ice-cold acetone. Cells were washed three times with PBS and then blocked in 1\% BSA. The fixed and permeabilized cells were incubated with $20 \mu \mathrm{g} / \mathrm{ml}$ goat anti-human BCL2 (R\&D Inc.) and $2.5 \mu \mathrm{g} / \mathrm{ml}$ mouse anti-gelsolin (BD Biosciences) at $22-25{ }^{\circ} \mathrm{C}$ for $1 \mathrm{~h}$. The cells were then washed three times with PBS, followed by a 40-min incubation with a 1:100 dilution of FITC-labeled anti-mouse IgG and Alexa 647-conjugated chicken anti-goat IgG (1:200 dilution). After washing four times with PBS, the cells were mounted on slides with Mountshield containing DAPI and imaged using a Zeiss LSM 510 NLO confocal/multiphoton laserscanning attachment (Carl Zeiss Inc., Thornwood, NY, USA) on the Zeiss Axiovert 100-M microscope. Three image acquisitions occurred sequentially with a C-Apo $40 \times / 1.2 \mathrm{~W}$ objective lens and optimized dichroics. For DAPI localization, two-photon excitation was used at $750 \mathrm{~nm}$, using a BP 435-485 emission filter. For FITC, the excitation wavelength was $488 \mathrm{~nm}$ and the BP 500-550 filter was used for emission. For Alexa Fluor 647, a 633-nm laser was used for excitation and an LP 650 filter for emission. For the two confocal channels, the pinhole was set to $\sim 1$ Airy Unit so that $\mathrm{z}$ resolution would match that of the two-photon excitation. The DIC image was acquired simultaneously with the FITC image. The $\mathrm{XY}$ alignment of the DAPI image was performed post acquisition using the DIC image as reference, and all channels were contrast stretched, using Zeiss LSM Image Examiner software.

The cells for F-actin staining were treated with $0.1 \%$ Triton $\mathrm{X}-100$ in PBS for $5 \mathrm{~min}$ after fixation as described above. Rhodamine phalloidin $(5 \mu \mathrm{l})$ from $6.6 \mathrm{M}$ stock solution was diluted into $200 \mu \mathrm{l}$ PBS and placed onto the coverslip for $20 \mathrm{~min}$ at $22-25^{\circ} \mathrm{C}$. Cells were then washed four times with PBS. Images and the intensity of fluorescence were obtained with an Olympus IX 70 fluorescence microscope (Olympus) and a DXC-S500 (Sony, Japan) color digital camera. Images were analyzed with Zeiss LSM Image examiner. Quantitative analysis of the images was performed with Zeiss LSM Image Examiner software. A background value was determined from the pixel intensity histogram of each image, taken as the statistical mode above zero. This value was subtracted from the mean pixel value for all pixels above a threshold determined by visual exclusion of background pixels. This threshold was used subsequently for all images. The background-subtracted 
mean pixel value was then multiplied by the pixel area to get total fluorescence intensity, and this number was divided by the number of cells to get the average fluorescence per cell, all in arbitrary fluorescence units.

(Experimental materials and methods are depicted in the Supplementary information, Data S1)

\section{Acknowledgments}

We thank Dr Jau-shyong Hong (NIEHS), Dr Bob Petrovich (NIEHS), Ms Pamela D Arora (CIHR, Toronto), and Dr Yanhong Liao (Huazhong University) for materials, Dr Jeff Chou (NIEHS) for statistics, and Dr James Williams (NIEHS) for mass spectra analysis. We also thank Dr Azad Bonni (Harvard Medical School) for providing plasmid-expressing GST-bad. The Division of Intramural Research of the NIEHS, NIH supported this research through projects Z01-ES02302511 and Z01-ES2120714.

\section{References}

1 Reed JC. Double identity for proteins of the Bcl-2 family. $\mathrm{Na}$ ture 1997; 387:773-776.

2 Strasser A, Harris AW, Cory S. E mu-bcl-2 transgene facilitates spontaneous transformation of early pre-B and immunoglobulin-secreting cells but not T cells. Oncogene 1993; 8:1-9.

3 Kumar P, Ning Y, Polverini PJ. Endothelial cells expressing Bcl-2 promotes tumor metastasis by enhancing tumor angiogenesis, blood vessel leakiness and tumor invasion. $L a b$ Invest 2008; 88:740-749.

4 Yilmaz A, Savas I, Dizbay Sak S, et al. Distribution of Bcl2 gene expression and its prognostic value in non-small cell lung cancer. Tuberk Toraks 2005; 53:323-329.

5 Itoi T, Yamana K, Bilim V, Takahashi K, Tomita F. Impact of frequent $\mathrm{Bcl}-2$ expression on better prognosis in renal cell carcinoma patients. Br J Cancer 2004; 90:200-205.

6 Leahy DT, Mulcahy HE, O’Donoghue DP, Parfrey NA. bcl-2 protein expression is associated with better prognosis in colorectal cancer. Histopathology 1999; 35:360-367.

7 Divito KA, Berger AJ, Camp RL, et al. Automated quantitative analysis of tissue microarrays reveals an association between high $\mathrm{Bcl}-2$ expression and improved outcome in melanoma. Cancer Res 2004; 64:8773-8777.

8 Neri A, Marrelli D, Roviello F, et al. Bcl-2 expression correlates with lymphovascular invasion and long-term prognosis in breast cancer. Breast Cancer Res Treat 2006; 99:77-83.

9 Rathmell JC, Thompson CB. Pathways of apoptosis in lymphocyte development, homeostasis, and disease. Cell 2002; 109 Suppl:S97-S107.

10 Akiyama SK, Olden K, Yamada KM. Fibronectin and integrins in invasion and metastasis. Cancer Metastasis Rev 1995; 14:173-189.

11 Bokel C, Brown NH. Integrins in development: moving on, responding to, and sticking to the extracellular matrix. Dev Cell 2002; 3:311-321.

12 Kedrin D, van Rheenen J, Hernandez L, Condeelis J, Segall JE. Cell motility and cytoskeletal regulation in invasion and metastasis. J Mammary Gland Biol Neoplasia 2007; 12:143152.

13 Ridley AJ, Schwartz MA, Burridge K, et al. Cell migra- tion: integrating signals from front to back. Science 2003; 302:1704-1709.

14 Webb DJ, Brown CM, Horwitz AF. Illuminating adhesion complexes in migrating cells: moving toward a bright future. Curr Opin Cell Biol 2003; 15:614-620.

15 Disanza A, Steffen A, Hertzog M, et al. Actin polymerization machinery: the finish line of signaling networks, the starting point of cellular movement. Cell Mol Life Sci 2005; 62:955970 .

16 Kassis J, Lauffenburger DA, Turner T, Wells A. Tumor invasion as dysregulated cell motility. Semin Cancer Biol 2001; 11:105-117.

17 Pantaloni D, Le Clainche C, Carlier MF. Mechanism of actinbased motility. Science 2001; 292:1502-1506.

18 Pollard TD. The cytoskeleton, cellular motility and the reductionist agenda. Nature 2003; 422:741-745.

19 Small JV, Stradal T, Vignal E, Rottner K. The lamellipodium: where motility begins. Trends Cell Biol 2002; 12:112-120.

20 Silacci P, Mazzolai L, Gauci C, et al. Gelsolin superfamily proteins: key regulators of cellular functions. Cell Mol Life Sci 2004; 61:2614-2623.

21 Linder S, Aepfelbacher M. Podosomes: adhesion hot-spots of invasive cells. Trends Cell Biol 2003; 13:376-385.

22 De Corte V, Bruyneel E, Boucherie C, et al. Gelsolin-induced epithelial cell invasion is dependent on Ras-Rac signaling. EMBO J 2002; 21:6781-6790.

23 Larson L, Arnaudeau S, Gibson B, et al. Gelsolin mediates calcium-dependent disassembly of Listeria actin tails. Proc Natl Acad Sci USA 2005; 102:1921-1926.

24 Azuma T, Witke W, Stossel TP, Hartwig JH, Kwiatkowski DJ. Gelsolin is a downstream effector of rac for fibroblast motility. EMBO J 1998; 17:1362-1370.

25 Cunningham CC, Stossel TP, Kwiatkowski DJ. Enhanced motility in NIH 3 T3 fibroblasts that overexpress gelsolin. Science 1991; 251:1233-1236.

26 Arora PD, McCulloch CA. Dependence of fibroblast migration on actin severing activity of gelsolin. J Biol Chem 1996; 271:20516-20523.

27 Akiyama SK. Functional analysis of cell adhesion: quantitation of cell-matrix attachment. Methods Cell Biol 2002; 69:281-296.

28 Gildea JJ, Harding MA, Gulding KM, Theodorescu D. Transmembrane motility assay of transiently transfected cells by fluorescent cell counting and luciferase measurement. Biotechniques 2000; 29:81-86.

29 Reinhart-King CA, Dembo M, Hammer DA. The dynamics and mechanics of endothelial cell spreading. Biophys $J$ 2005; 89:676-689.

30 Apostolova MD, Christova T, Templeton DM. Involvement of gelsolin in cadmium-induced disruption of the mesangial cell cytoskeleton. Toxicol Sci 2006; 89:465-474.

31 Li S, Guan JL, Chien S. Biochemistry and biomechanics of cell motility. Annu Rev Biomed Eng 2005; 7:105-150.

32 Zhang W, Pantschenko AG, McCarthy MB, Gronowicz G. Bone-targeted overexpression of Bcl-2 increases osteoblast adhesion and differentiation and inhibits mineralization in vitro. Calcif Tissue Int 2007; 80:111-122.

33 Sheibani N, Scheef EA, Dimaio TA, et al. Bcl-2 expression modulates cell adhesion and migration promoting branching 
of ureteric bud cells. J Cell Physiol 2007; 210:616-625.

34 Lambrechts A, Van Troys M, Ampe C. The actin cytoskeleton in normal and pathological cell motility. Int J Biochem Cell Biol 2004; 36:1890-1909.

35 Moissoglu K, Schwartz MA. Integrin signalling in directed cell migration. Biol Cell 2006; 98:547-555.

36 Suetsugu S, Takenawa T. Regulation of cortical actin networks in cell migration. Int Rev Cytol 2003; 2229:245-286.

37 Lauffenburger DA, Horwitz AF. Cell migration: a physically integrated molecular process. Cell 1996; 84:359-369.

38 Howe A, Aplin AE, Alahari SK, Juliano RL. Integrin signaling and cell growth control. Curr Opin Cell Biol 1998; 10:220-231.

39 Hynes RO. Integrins: bidirectional, allosteric signaling machines. Cell 2002; 110:673-687.

40 Huttenlocher A, Sandborg RR, Horwitz AF. Adhesion in cell migration. Curr Opin Cell Biol 1995; 7:697-706.

41 Ruoslahti E. Fibronectin and its integrin receptors in cancer. Adv Cancer Res 1999; 76:1-20.

42 Schwartz MA, Schaller MD, Ginsberg MH. Integrins: emerging paradigms of signal transduction. Annu Rev Cell Dev Biol 1995; 11:549-599.

43 Feldner JC, Brandt BH. Cancer cell motility - on the road from c-erbB-2 receptor steered signaling to actin reorganization. Exp Cell Res 2002; 272:93-108.

44 Zigmond SH. Beginning and ending an actin filament: control at the barbed end. Curr Top Dev Biol 2004; 63:145-188.

45 Chou J, Stolz DB, Burke NA, Watkins SC, Wells A. Distribution of gelsolin and phosphoinositol 4,5-bisphosphate in lamellipodia during EGF-induced motility. Int J Biochem Cell Biol 2002; 34:776-790.

46 Janmey PA, Stossel TP. Gelsolin-polyphosphoinositide interaction. Full expression of gelsolin-inhibiting function by polyphosphoinositides in vesicular form and inactivation by dilution, aggregation, or masking of the inositol head group. $J$ Biol Chem 1989; 264:4825-4831.

47 Mere J, Chahinian A, Maciver SK, et al. Gelsolin binds to polyphosphoinositide-free lipid vesicles and simultaneously to actin microfilaments. Biochem J 2005; 386 (Part 1):47-56.

48 Chellaiah M, Hruska K. Osteopontin stimulates gelsolin- associated phosphoinositide levels and phosphatidylinositol triphosphate-hydroxyl kinase. Mol Biol Cell 1996; 7:743-753.

49 Ziehr J, Sheibani N, Sorenson CM. Alterations in celladhesive and migratory properties of proximal tubule and collecting duct cells from bcl-2 -/- mice. Am J Physiol Renal Physiol 2004; 287:F1154-F1163.

50 Sorenson CM. Interaction of bcl-2 with Paxillin through its BH4 domain is important during ureteric bud branching. $J$ Biol Chem 2004; 279:11368-11374.

51 Chellaiah MA, Biswas RS, Yuen D, Alvarez UM, Hruska KA. Phosphatidylinositol 3,4,5-trisphosphate directs association of Src homology 2-containing signaling proteins with gelsolin. $J$ Biol Chem 2001; 276:47434-47444.

52 Sorenson CM, Sheibani N. Focal adhesion kinase, paxillin, and bcl-2: analysis of expression, phosphorylation, and association during morphogenesis. Dev Dyn 1999; 215:371-382.

53 Callagy GM, Webber MJ, Pharoah PD, Caldas C. Metaanalysis confirms BCL2 is an independent prognostic marker in breast cancer. BMC Cancer 2008; 8:153.

54 Monni O, Joensuu H, Franssila K, et al. BCL2 overexpression associated with chromosomal amplification in diffuse large Bcell lymphoma. Blood 1997; 90:1168-1174.

55 Pinkas J, Martin SS, Leder P. Bcl-2-mediated cell survival promotes metastasis of EpH4 betaMEKDD mammary epithelial cells. Mol Cancer Res 2004; 2:551-556.

56 Ishido M, Ohtsubo R, Adachi T, Kunimoto M. Attenuation of both apoptotic and necrotic actions of cadmium by Bcl-2. Environ Health Perspect 2002; 110:37-42.

57 Martin SS, Leder P. Human MCF10A mammary epithelial cells undergo apoptosis following actin depolymerization that is independent of attachment and rescued by Bcl-2. Mol Cell Biol 2001; 21:6529-6536.

58 Lipton A, Klinger I, Paul D, Holley RW. Migration of mouse 3T3 fibroblasts in response to a serum factor. Proc Natl Acad Sci USA 1971; 68:2799-2801.

59 Allen PG, Janmey PA. Gelsolin displaces phalloidin from actin filaments. A new fluorescence method shows that both $\mathrm{Ca}^{2+}$ and $\mathrm{Mg}^{2+}$ affect the rate at which gelsolin severs F-actin. J Biol Chem 1994; 269:32916-32923.

(Supplementary information is linked to the online version of the paper on the Cell Research website.) 